\title{
MHEALTH MONITORING: REGISTRO Y MONITOREO DE PACIENTES EN UNA UNIDAD DE CUIDADOS INTENSIVOS NEONATALES USANDO LAS PLATAFORMAS MÓVIL Y WEB
}

\author{
MHEALTH MONITORING: REGISTRATION AND MONITORING \\ OF PATIENTS IN AN INTENSIVE CARE UNIT NEONATALS USING MOBILE ANDWEB PLATFORMS
}

Edgardo Palza ', Rosario Guzmán ², Hernan Rodríguez ${ }^{1}$

\begin{abstract}
RESUMEN
Objetivos: Una Unidad de Cuidados Intensivos Neonatales (UCIN) tiene como misión brindar atención médica a partos complejos y recién nacidos con algún grado de enfermedad: en muchos casos estos pacientes necesitan atención médica especializada y monitoreo. El presente artículo muestra el análisis, diseño, desarrollo e implementación de un sistema dei nformación de salud basado en plataformas web y móviles que cumplen con los requerimientos de una UCIN. Métodos: El sistema se ha desarrollado como una aplicación de BPM (Business Process Management - Gestión de Procesos de Negocios) y BPMN (Business Process Modeling Notation - Notación de Modelado de Procesos de Negocios) y proporciona al personal médico y de enfermería la capacidad de registrar y supervisar los resultados de los tratamientos médicos, pruebas de laboratorio, medicamentos recetados, cálculo de dosis de medicamentos a los pacientes, etc. Resultados: La aplicación de BPM / BPMN ofrece el monitoreo y control de diferentes procesos y recursos dentro de la UCIN. El software también proporciona KPI (Key Performance Indicators - Indicadores Clave de Rendimiento) para pacientes, personal y otros recursos a través de tecnologías web y móviles. Este trabajo se llevó a cabo con la colaboración de un hospital en Perú. Conclusión: El proyecto fue diseñado e implementado con la solución "IBM Business Process Manager". La solución mHealth-BPM-UCIN está en un prototipo y una fase de prueba en la UCIN.
\end{abstract}

Palabras clave: ABPM; BPMN; KPI; UCIN; Salud Web; Salud Móvil; Sistema de Información de Salud; Metodología BPM (fuente: DeCS BIREME).

\section{ABSTRACT}

Objective: A Neonatal Intensive Care Unit (NICU) is usually part of the Emergency Department and its mission is to provide healthcare to complex births and sickest newborns: in many cases these patients need specialized medical care and monitoring. This chapter presents an overview of the analysis, design, development and implementation of a Health Information System based on Web and Mobile technologies that meets the requirements of a NICU. Methods: The system has been developed as a BPM/BPMN application and provides the medical and nursing staff the ability to record and monitor the results of the medical treatments, laboratory tests, prescribed drugs, calculation of drug doses to patients, and others features. Results: The BPM/BPMN application offers monitoring and controlling different processes and resources inside the NICU. The software also provides Key Performance Indicators (KPI) for patients, staff and other resources through Web and mobile technologies. This work was carried on with the collaboration of a hospital in Peru. Conclusion: The project was designed and implemented with the "IBM Business Process Manager" solution. The mHealth-BPM-UCIN solution which is in a prototype and a test phase at the NICU.

Key words: BPM; BPMN; KPI; NICU; Web Health; Mobile Health; Health Information System; BPM Methodology (source: MeSH NLM).

\footnotetext{
1 Universidad ESAN, Perú.
}

2Universidad de Lima, Perú.

Correspondencia: Facultad de Ingeniería, Universidad ESAN. Dirección: Alonso de molina 1652, Monterrico, surco, Lima-Perú. Teléfono: (511) 317-7200 Anexo: 4378. Correo : epalzaesan.edu.pe

Citar como: Edgardo Palza, Rosario Guzmán, Hernán Rodríguez. Mhealth Monitoring: Registro y Monitoreo de Pacientes en una Unidad de Cuidados Intensivos Neonatales Usando las Plataformas Móvil y Web [Artículo Original]. Rev. Fac. Med. Hum. 2016;16(2):18-30. DOI 10.25176/RFMH.v16.n2.664 


\section{INTRODUCCIÓN}

Una Unidad de Cuidados Intensivos Neonatales (UCIN) generalmente es parte del Departamento de Emergencias y su misión es proporcionar atención de alta complejidad a pacientes prematuros o de término, con patologías que pueden tener complicaciones que requieren investigación, manejo y monitoreo especializado. La UCIN debe contar con personal adecuado, un equipo de profesionales capacitados para brindar atención especializada a los recién nacidos.

Es bien sabido que muchas veces los hospitales ofrecen servicios de salud con recursos limitados en cuanto a infraestructura, profesionales, etc. Además, las áreas de emergencia y hospitalización a menudo están sobrecargadas de pacientes, lo que dificulta la administración de los servicios de salud.

Particularmente los problemas en la administración de servicios se muestra más evidente en la UCIN donde la identificación de eventos adversos en la evolución del recién nacido es vital: se necesita información precisa y oportuna para permitir que el médico o la enfermera determinen el nivel de riesgo del paciente en cualquier momento. Desafortunadamente en muchos hospitales la realidad es diferente: la falta de acceso a la información histórica integrada, consistente y confiable deteriora a veces el diagnóstico médico adecuado y asertivo.

En la mayoría de los hospitales, todo el proceso de atención se registra en papel, que con el tiempo puede ser dañado; Además, la información es a menudo incompleta, incorrecta o ininteligible para la interpretación. El crecimiento incontrolado de los pacientes hospitalizados también está agravando estos problemas. En tales contextos es muy difícil asignar adecuadamente personal, medicamentos y equipo médico a la Unidad y controlar y medir la efectividad y eficiencia de la UCIN.

El objetivo del presente proyecto fue diseñar un Sistema de Información basado en una plataforma Web y móvil que permita la mejora y automatización de procesos en la UCIN con la capacidad de registrar y monitorear lo siguiente:

- Los resultados de procedimientos.

- Los tratamientos médicos

- Las evaluaciones de laboratorio

- Los medicamentos prescritos

- La información básica sobre la madre

- Las condiciones de parto promedio

- El seguimiento de los resultados de los pacientes en la unidad

- El cálculo de dosis de fármaco a los pacientes
- La aprobacióny la asignación de personal a los pacientes

- El número de pacientes tratados

- La duración de la estancia

Este artículo presenta el diseño y despliegue de una solución de mHealth diseñada con en la plataforma BPM (Business Process Management) ${ }^{1}$ y la notación BPMN (Business Process Model and Notation) ${ }^{7}$, utilizando tecnologías Web y Móviles.

\section{TRABAJOS RELACIONADOS}

Se explica ${ }^{2}$ que, dados los desafíos impuestos en el contexto de la Administración Hospitalaria, surgieron conceptos como "HPM (Healthcare Process Management - Gestión de Procesos de Salud)". El HPM utiliza las mejores prácticas de Gestión de Procesos de Negocio (BPM) para gestionar la asignación de servicios considerando la complejidad inherente de los hospitales. Los autores afirman que BPM puede ayudar a optimizar los esfuerzos y mejorar el tiempo de los servicios, así como desarrollar la visibilidad de los procesos de salud y el rendimiento en el hospital.

Se ha presentado ${ }^{4}$ una solución para la recolección, análisis y reporte de datos de diferentes fuentes dentro de una entidad de salud. Para abordar los problemas comunes a los que se enfrentan las organizaciones en términos de registro, seguimiento y control de los pacientes, proponen una plataforma para integrar diferentes fuentes con el fin de facilitar la disponibilidad y el acceso de los datos por parte de los profesionales. Su plataforma propuesta brinda un acceso en tiempo real a los datos.

Se muestra ${ }^{8}$ la implementación de un sistema de información integrado para la sala de urgencias de un hospital en Portugal. La solución propuesta captura la información clínica del paciente, como signos vitales, ventilación, medicación, etc., directamente del equipo médico. También se discuten las mejoras logradas en términos de calidad general del servicio en la sala.

Se resenta ${ }^{5}$ el reto de capturar datos de la evolución fisiológica y médica de los recién nacidos en una UCIN. Proponen una solución completa de inteligencia de negocios que recopila datos de los dispositivos médicos en una UCIN. Los autores proponen una solución modular y en capas que permite la visualización de los datos contenidos en los repositorios previamente establecidos. Su solución ha sido implementada como prototipo en el "Nepean Hospital" de Australia.

Se desarrolló 9 una aplicación web para facilitar el control y monitoreo de las condiciones de los recién nacidos en la UCIN. Esta aplicación permite hacer un seguimiento de los tratamientos y medicamentos del paciente, así como registrar la información de las pruebas de labora- 
torio, evaluaciones, incluyendo imágenes de los rayos $\mathrm{X}$ y electrocardiogramas. La aplicación web también tiene la capacidad de consolidar los resultados y los informes según sea necesario. La solución se construye utilizando una tecnología SOA (Service Oriented Architecture - Arquitectura Orientada a los Servicios) que permite la adición de datos recopilados de otras plataformas.

\section{METODOLOGÍA DE DESARROLLO DE BPM}

La metodología "Playback"3,10 fue seleccionada como metodología de BPM para implementar mejoras y gestionar los procesos de negocio de nuestro proyecto. "Playback" Se basa en un proceso de despliegue iterativo (Figura 1), estos procesos se modelan utilizando la notación de BPMN. La primera versión, denominada "Playback 0", se inicia después de haber identificado el proceso para ser desarrollado.

La siguiente versión, Playback 1, tiene su propia actividad en la primera versión del proceso y se puede dividir en dos etapas (1A y 1B), detallando las actividades y las tareas a realizar. El "Playback $1 b^{\prime \prime}$ se centra en la creación y personalización de interfaces de usuario de la aplicación.

El "Playback 2" implementa la integración con sistemas externos, así como servicios de decisión y procesos externos.

El “Playback 3” se centra en la mejora de las interfaces de usuario, la definición de informes para supervisar el proceso y la aplicación prudente de la gestión de errores.
Para cada lanzamiento (o playback) la recolección de requisitos nunca se detiene. Los requerimientos se refinan a través de una serie de reproducciones y cada reproducción es una planificación y despliegue de iteración (Figura 1). Por ejemplo, se pueden agregar nuevos requisitos en una nueva sesión de usuario y se pueden reproducir en la próxima versión.

\section{DISEÑO Y DESARROLLO DE LA SOLUCIÓN}

El análisis, diseño e implementación de la solución se llevó a cabo de la siguiente manera.

\section{Primera etapa: " Playback 0" - Definir los procesos "As-Is" ("Como es")}

\section{Admisión y diagnóstico}

El personal médico en servicio evalúa al paciente y lo registra en la hoja de admisión (Ilamada "Epicrisis"), mientras que simultáneamente se completa un primer diagnóstico del recién nacido. Además, el médico debe llenar una hoja de tratamiento, que contiene la dosis de fármaco respectiva aplicada al paciente, así como el plan de alimentación.

Los criterios para ingresar a un recién nacido en la UCIN están regulados por el MINSA $^{6}$ y entre otros ellos son: Recién nacido prematuro, angustia respiratoria neonatal, asfixia de nacimiento, taquipnea transitoria del recién nacido, aspiración del síndrome neonatal, enfermedad hemolítica neonatal y del feto, ictericia neonatal por hemólisis excesiva, ictericia neonatal por causas no especificadas, y otras.

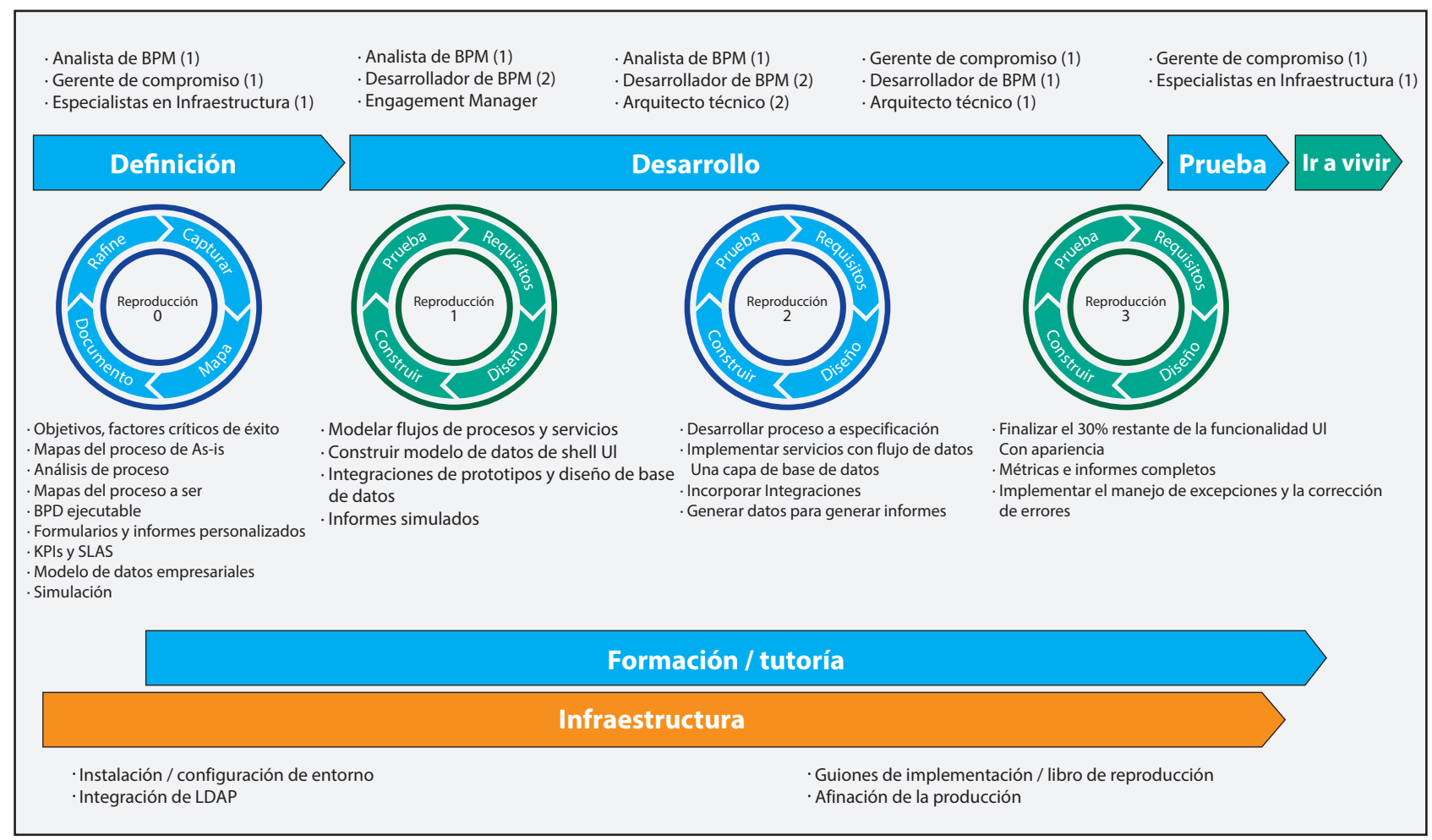

Figura 1. Metodología de"Playback"11. 
Según entrevistas con algunos médicos en la UCIN, cada bebé es evaluado individualmente para determinar el requisito de admisión a la unidad. Sin embargo, un gran porcentaje de recién nacidos son admitidos porque son prematuros: significa que nacen antes de las 37 semanas de embarazo o porque tienen bajo peso al nacer (menos de 2.250 gramos o 5,5 libras), o porque padecen una afección médica que requiere atención médica especial. Por otra parte, los nacimientos de embarazos múltiples (gemelos, trillizos, o más) son a menudo admitidos en la UCIN por tener bajo peso y un tamaño menor al normal de un recién nacido.

\section{Seguimiento y lanzamiento}

Las enfermeras preparan su programa personalizado de trabajo diario para cada paciente asignado sobre la base de las indicaciones de los médicos. De acuerdo con las regulaciones del Ministerio de Salud ${ }^{6}$ a cada enfermera se le debe asignar entre 1 y 2 pacientes a cargo, pero debido a la alta demanda deben aceptan más.

Como se indica en las hojas de tratamiento, las enfermeras deben realizar procedimientos de monitoreo a cada paciente; esto implica la evaluación diaria de pacientes (signos vitales), administración de medicamentos, registro de dosis, alimentación, análisis de los principales aspectos de la evolución del recién nacido, estudio de electrolitos del paciente, etc.

Para determinar la entrega del fármaco, las enfermeras tienen que realizar cálculos manuales a fin de determinar qué cantidad debe asignarse en función de los períodos de tiempo y lo cual que se especifica en las hojas de tratamiento.

\section{Registro manual}

Todos los registros y seguimientos de neonatos en la UCIN se realizan manualmente y en papel (formato identificado por un número de expediente médico). Por ejemplo, si un recién nacido es admitido por primera vez, la información requerida es: la información personal de la madre y el niño, los exámenes médicos, el informe de un diagnóstico médico, el tratamiento recomendado, las observaciones generales del médico y enfermeras a cargo y otros.

Deben crear números de historia manualmente y si la paciente se reintegra al servicio, la madre tiene que indicar el número de registro y el nombre del bebé, para que puedan localizar los registros médicos almacenados en los archivos (figura 2).

\section{Problemas observados}

Como ya se mencionó, uno de los problemas más críticos en la UCIN es que la gestión se realiza manualmente y el uso de registros en papel causa varios tipos de problemas: errores en el cálculo de las dosis de medicamento a los pacientes, errores en el llenado de los formularios (ilegible, etc.), daños a ellos ya sea por manipulación o envejecimiento, pérdida de documentos, etc.

Además de los problemas identificados, hay otros como el retraso en el alta del paciente, que en muchos casos también se debe a las limitadas condiciones de la Unidad. Por otro lado, es importante dejar claro que el crecimiento descontrolado en el número de pacientes en la unidad es factor que complica la realidad.

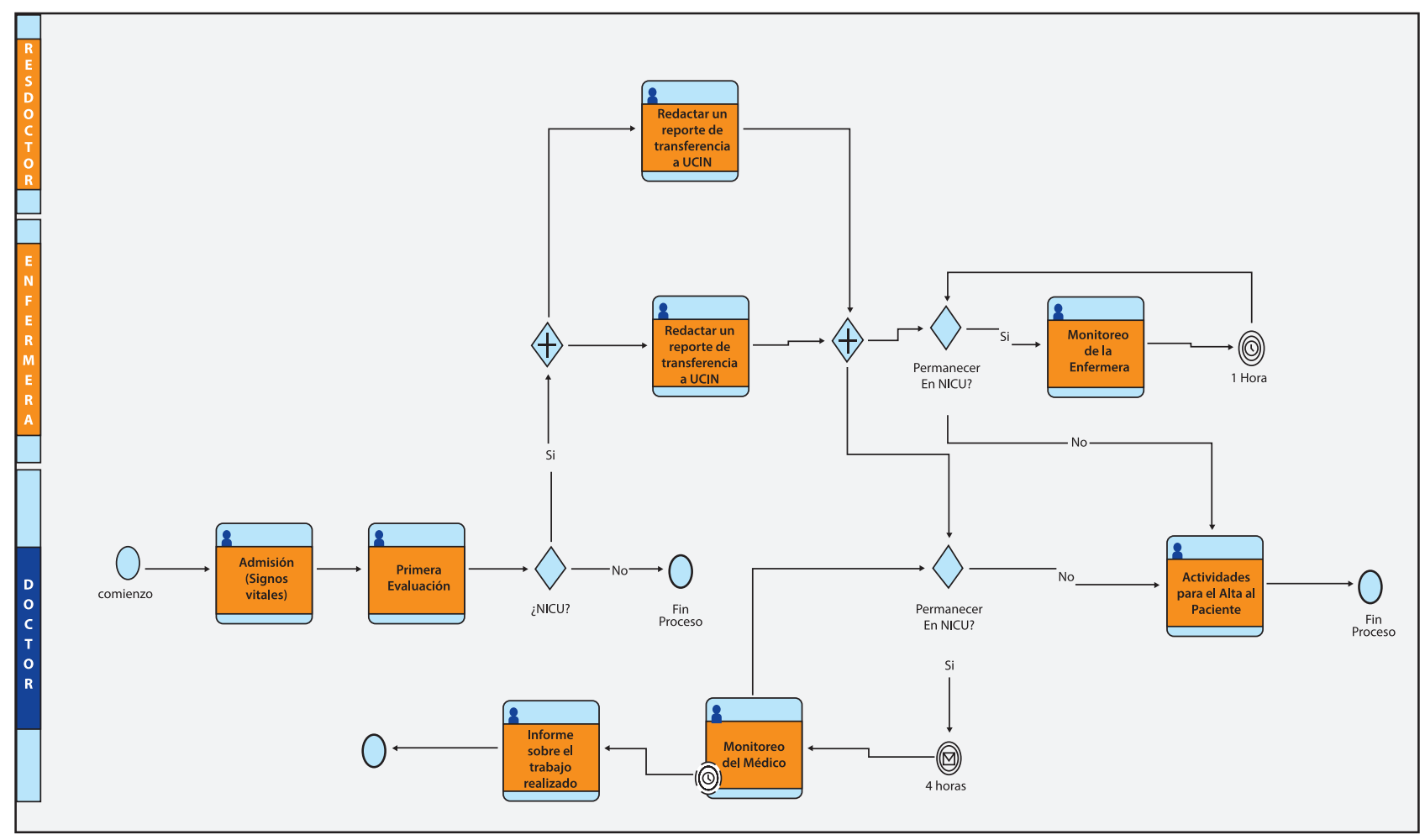

Figura 2. Procesos de "como están" en UCIN. 


\section{Segunda etapa: "Reproducción 1" - Creación del proceso "As-To be"}

La segunda etapa consiste en proponer procesos para mejorar, estandarizar y automatizar las mejores prácticas y documentos dentro de la UCIN. Los diagramas de procesos son la base para construir la solución de aplicación mHealth-BPM. Todas las interfaces, controles, indicadores, cálculos, conexiones de bases de datos y otros se implementarán en la aplicación basada en el diagrama BPMN. La arquitectura de la aplicación BPM se describirá en el próximo Playback.

La aplicación mHealth-BPM debe tener la capacidad de generar reportes de interés tales como: La historia clínica completa de cada recién nacido presentado diagnósticos, frecuencia de la enfermedad y tratamiento proporcionado, estas informaciones básicas apoyan la toma de decisiones por parte del Departamento de Neonatología.

En la aplicación mHealth-BPM, se establecen diferentes niveles de acceso, y todo el acceso a la información del neonato debe estar asegurado por nombres de usuario y contraseñas: los datos no deben mostrarse a usuarios no autorizados, los cambios y actualizaciones deben registrarse en el sistema de registro. El diagrama (figura 3 ) presenta la secuencia de procesos dentro de la solución propuesta de mHealth. Cada uno se describe a continuación

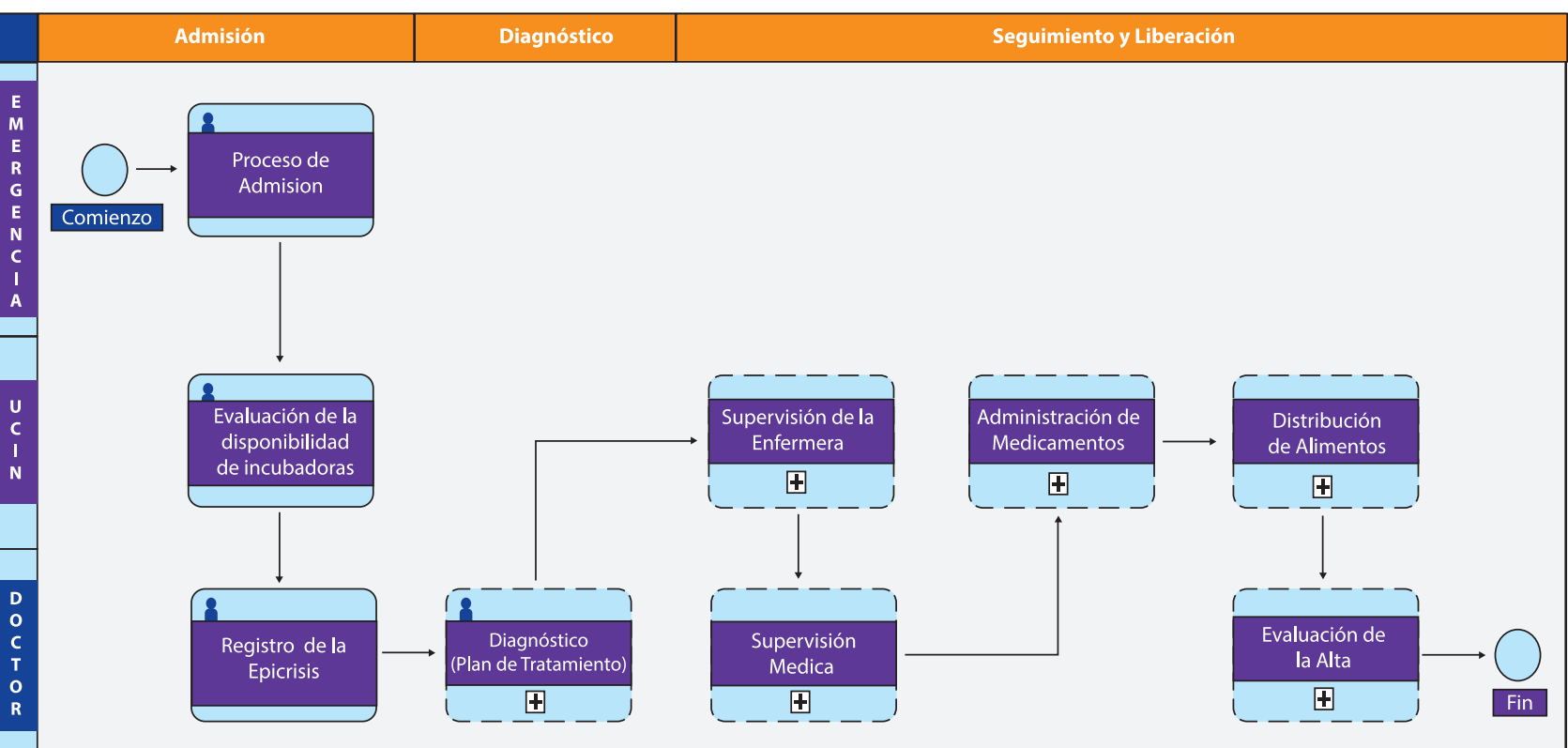

Figura 3. Procesos "As-Tobe" en la UCIN.

\section{Proceso de admisiones}

El proceso de admisión se compone de los siguientes tres subprocesos.

\section{Admisión de pacientes}

La admisión a la Unidad de Cuidados Intensivos de Neonatología (UCIN) puede ser generada desde la Sala de Entrega o la Unidad de Emergencia que generalmente es firmada UCIN. De acuerdo con la evaluación realizada por los médicos encargados de estas Unidades, pueden solicitar una transferencia a la UCIN a través de una hoja de transferencia entre las áreas involucradas.

La Figura 4 muestra una interfaz de teléfono inteligente implementada para registrar la admisión del paciente. Esta interfaz también podría abrirse en una computadora de escritorio.
- Buscando un paciente

En esta opción los médicos y enfermeras tienen la posibilidad de buscar diferentes opciones como: Nombres, fecha de admisión, expediente médico, fecha de nacimiento. Las figuras 5 y 6 muestran la implementación de la interfaz web y móvil.

\section{Evaluación de la disponibilidad de incubadoras}

Una vez realizada la coordinación entre unidades, la transferencia del paciente depende de la disponibilidad de incubadoras. Para la transferencia existen protocolos definidos por el Ministerio de Salud: en cada caso el paciente debe estar acompañado por una enfermera con historia clínica del paciente. 
$22^{*}$

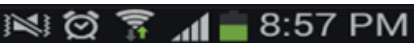

192.168.1.101:9080/teamworks 2 :

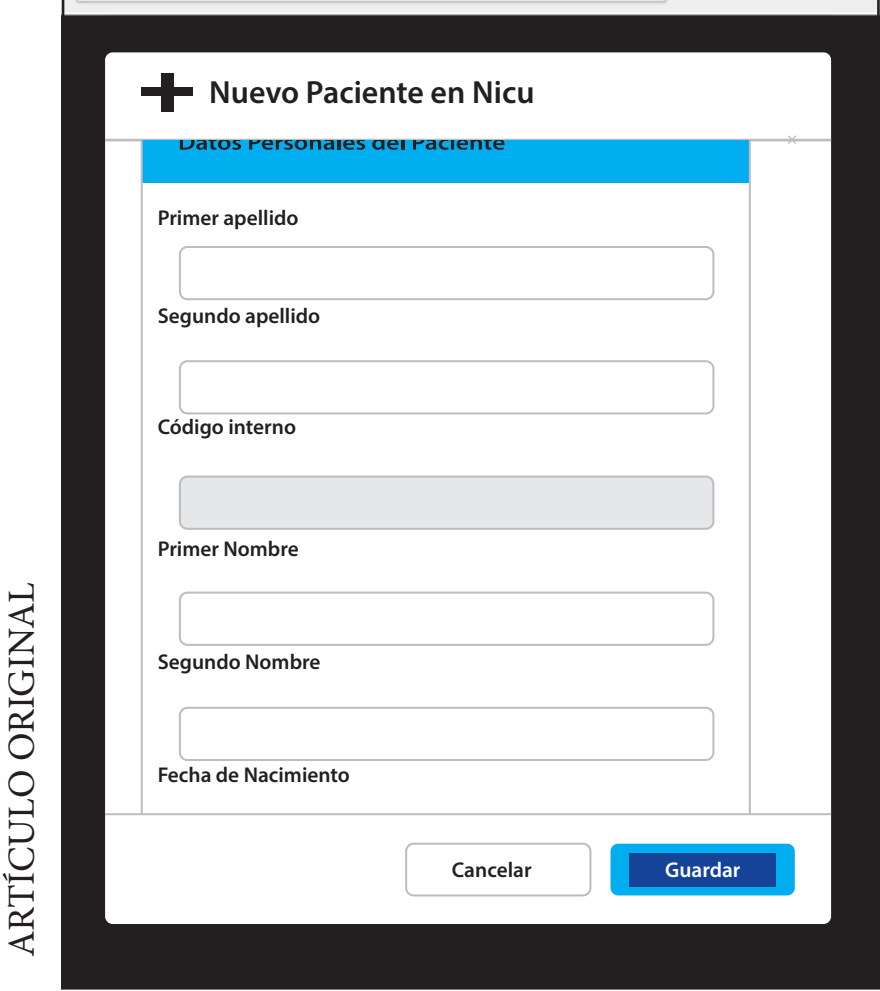

Figura 4. Registro de un nuevo paciente (plataforma móvil). $22^{\circ}$ 서

192.168.1.101:9080/teamworks 3 :

Resultado de búsqueda

\begin{tabular}{|c|c|c|c|c|}
\hline $\begin{array}{l}\text { Último } \\
\text { Nombre }\end{array}$ & Nombre & $\begin{array}{l}\text { Médico } \\
\text { Grabar }\end{array}$ & $\begin{array}{l}\text { Fecha de } \\
\text { admision } \\
\text { En NICU }\end{array}$ & $\begin{array}{l}\text { Fecha de } \\
\text { lanzamiento } \\
\text { En NICU }\end{array}$ \\
\hline \multirow[t]{2}{*}{ Lopez } & Martin & 13321 & $\begin{array}{c}\text { Jue Abril } \\
042013 \\
\text { 21:01:15 } \\
\text { GMT- } \\
0500 \\
\text { (PET) }\end{array}$ & $\begin{array}{c}\text { Sáb Abril } \\
\text { 06 2013 } \\
\text { 21:01:15 } \\
\text { GMT- } \\
0500 \\
\text { (PET) }\end{array}$ \\
\hline & María & 09269 & $\begin{array}{c}\text { Mie Enero } \\
282015 \\
21: 01: 15 \\
\text { GMT- } \\
0500 \\
\text { (PET) }\end{array}$ & \\
\hline
\end{tabular}

Mostrando 1 a 2 de 2 entradas

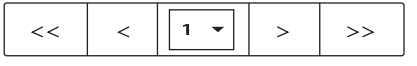

Figura 6. Búsqueda de un paciente (plataforma móvil).

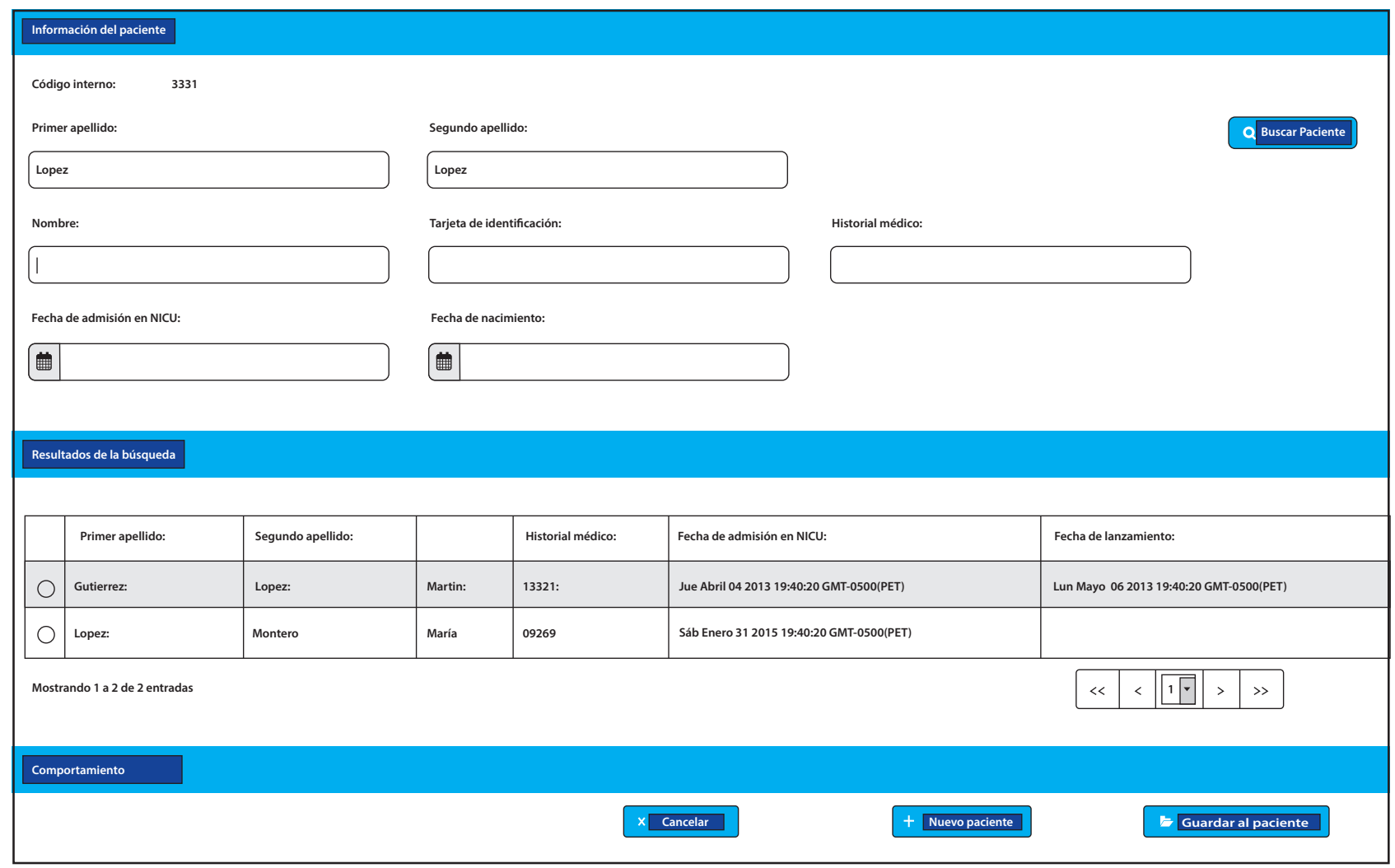

Figura 5. Búsqueda de un paciente (plataforma meb).

\section{Registro epicrisis}

Una vez que el paciente ha sido recibido en la la UCIN y se valida toda la información del expeUnidad, un médico registra la entrada formal a diente médico (Epicrisis) del paciente (figura 7). 


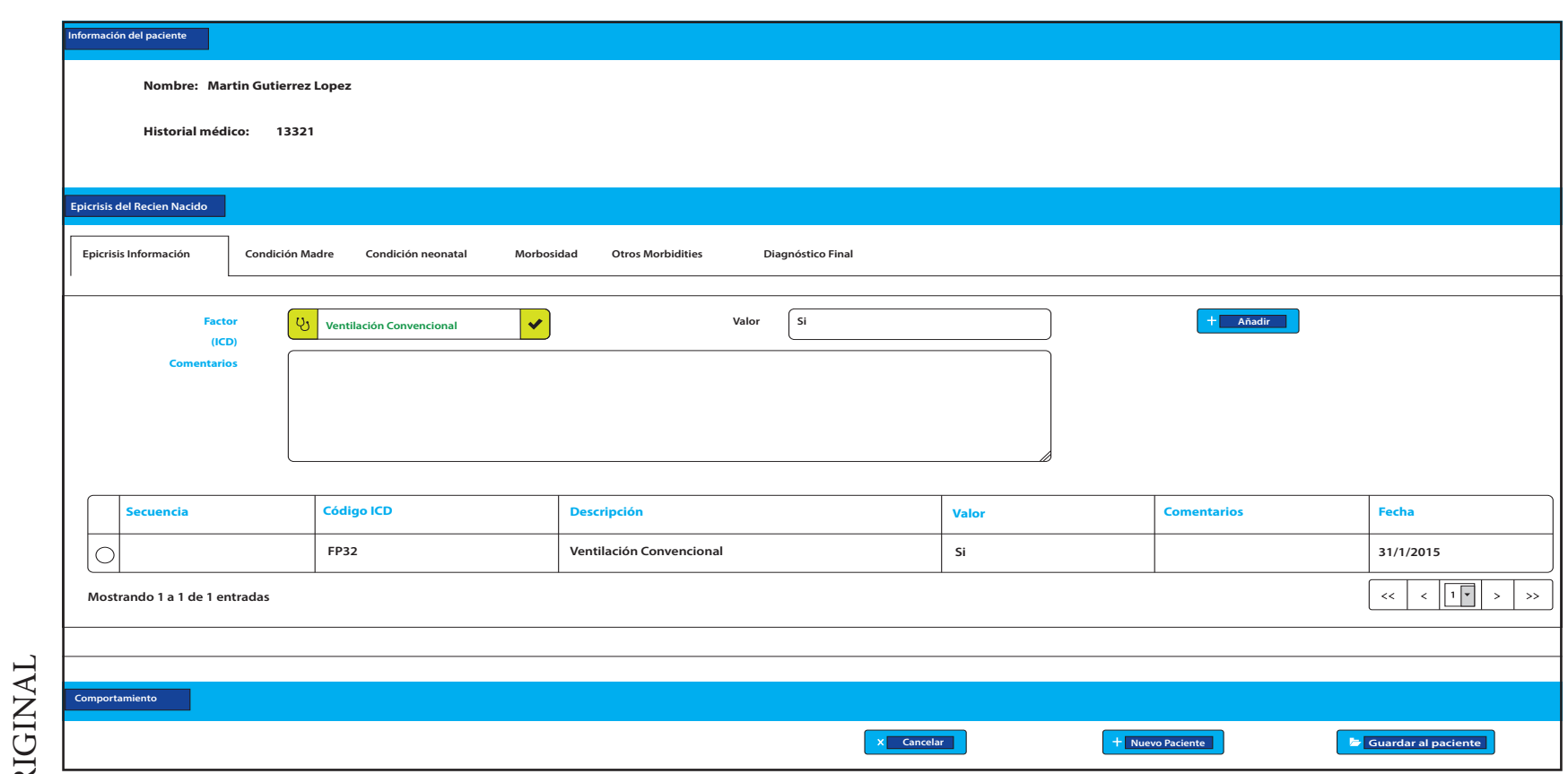

Figura 7. Primera evaluación y diagnóstico

\section{Diagnóstico}

Tratamiento propuesto

Una vez que el paciente está registrado, el médico evalúa la condición del paciente, revisa toda la documentación presentada y, sobre la base de esta información, indica el tratamiento a seguir: esto se registra en una hoja de evaluación y tratamiento y se adjunta toda la documentación relacionada con el paciente Al expediente médico, (figura 8).

- Evaluar los diagnósticos

Revisa la información del paciente tal como su historial médico.

- Calcular indicadores

Calcula automáticamente el equilibrio hídrico y la energía basada en el historial de datos.

\section{- Evaluación de medicamentos}

Permite consultar la base de datos de inventario de medicamentos registrados.

\section{- Registro de Tratamiento}

Registra el tratamiento que se ofrecerá al paciente en un período determinado.

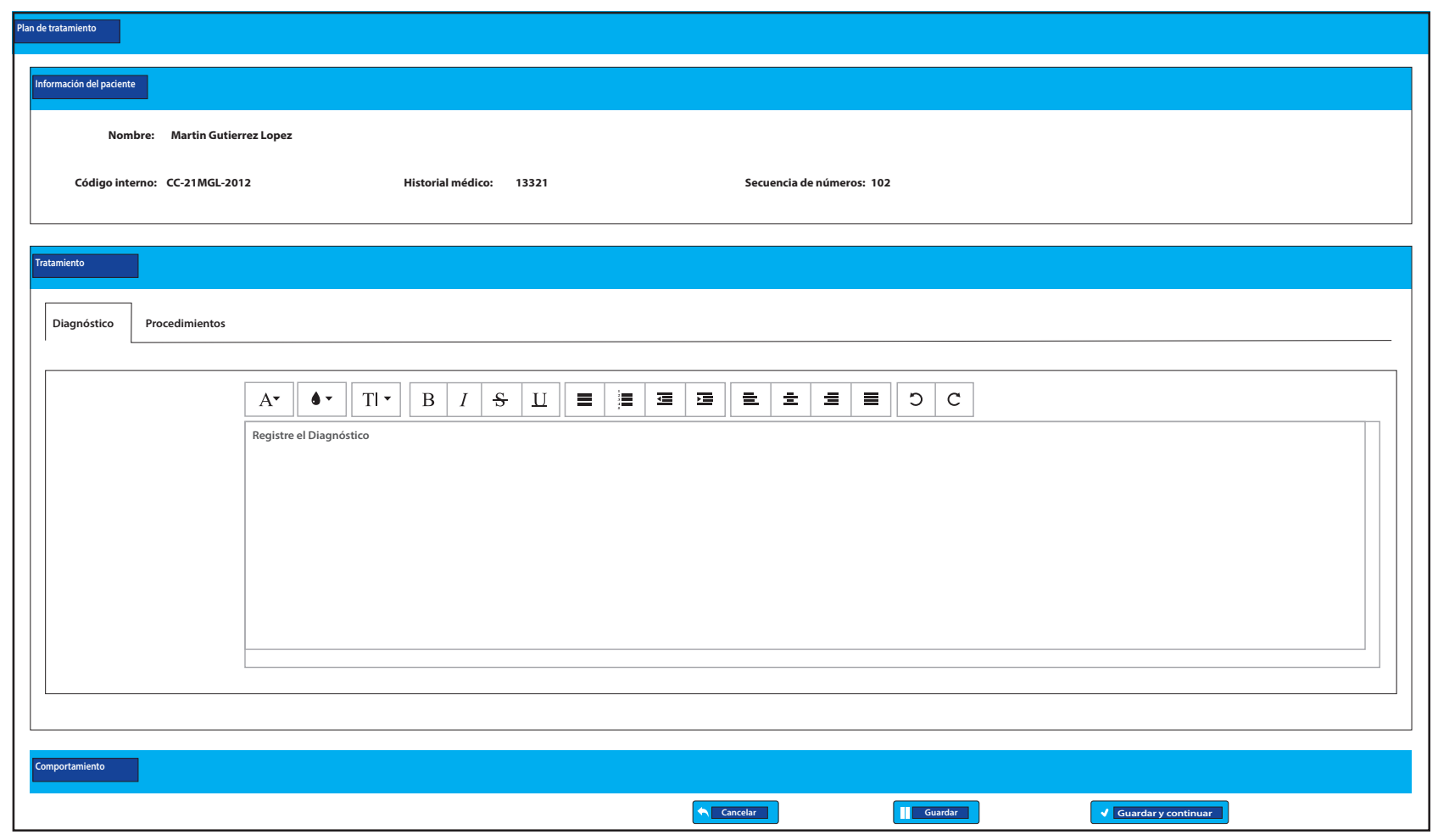

Figura 8. Plan de tratamiento. 


\section{Seguimiento y lanzamiento}

El proceso de monitoreo y liberación se compone de los siguientes subprocesos.

\section{Supervisión de enfermería}

Este proceso registra las actividades diarias según el protocolo establecido.

- Registre valores de signos vitales

Este es un procedimiento regulado bajo un protocolo del Ministerio de Salud. La frecuencia de este procedimiento depende de la crítica del paciente, pero por lo general se produce cada hora (Figura 9).

- Comparar los registros con los estándares

Para esta actividad, hay umbrales para cada uno de los signos vitales evaluados. Si hay algún comportamiento fuera del rango establecido, se procede a notar tanto la enfermera jefe y el médico de turno o el médico a cargo.

\section{Monitoreo médico}

Este proceso registra las actividades diarias para un paciente de acuerdo con una Hoja de Monitoreo del Protocolo.

\section{- Evaluación de registros}

El médico, después de leer el diagnóstico previo y el seguimiento de una historia del paciente, debe registrar su propia evaluación médica. La frecuencia de la evaluación depende de la criticidad del paciente, pero por lo general es cada 4 horas.

- Actualización del tratamiento de registro

El médico, basado en su propia evaluación y experiencia, debe determinar si continuar con el tratamiento establecido o indica el cambio o actualización.

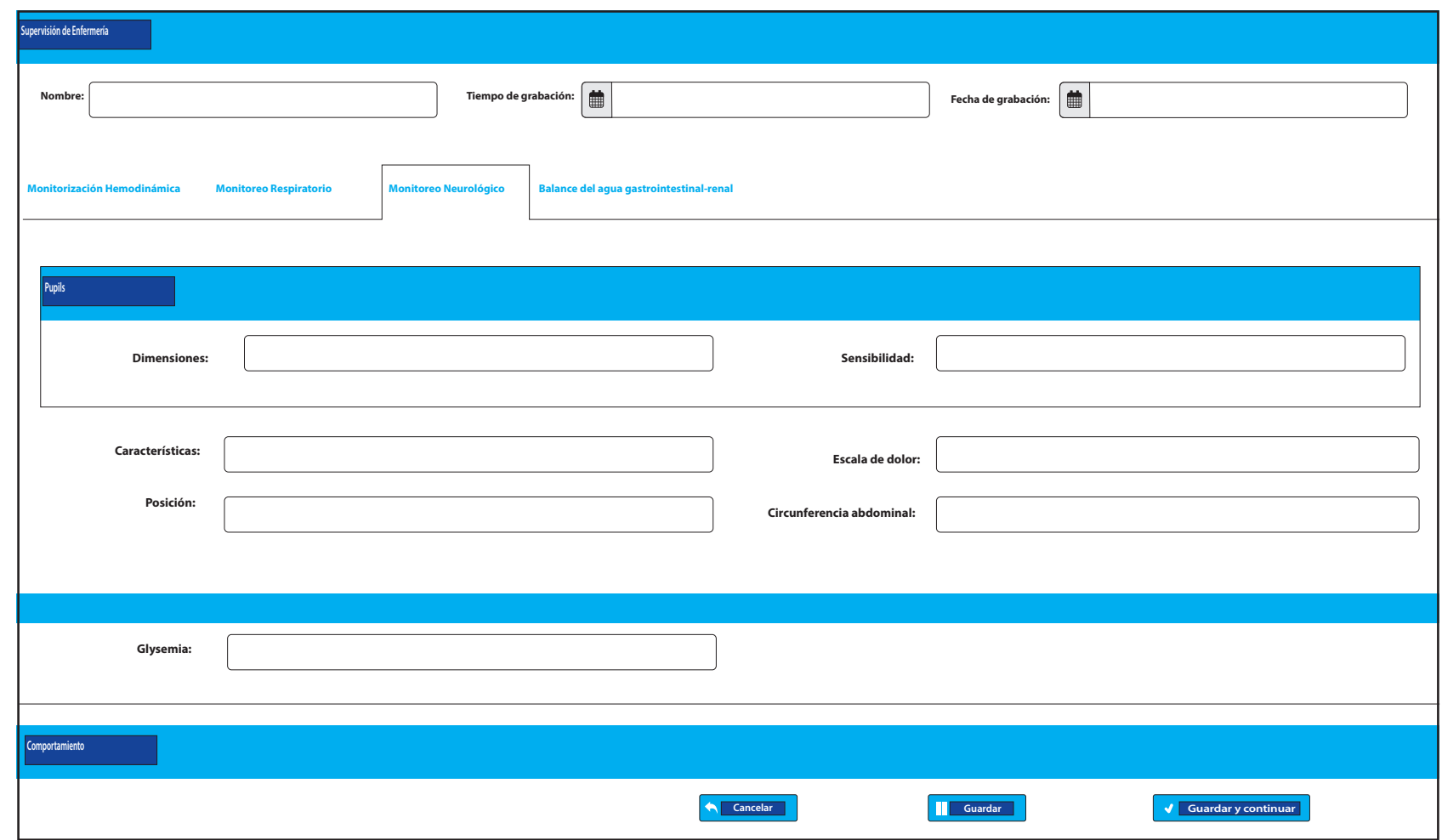

Figura 9. Signos vitales del paciente.

\section{Suministro de medicamentos}

Este proceso registra la dosificación del medicamento en una hoja de monitoreo: debe seguir las indicaciones del médico.

- Leer registro de tratamiento

Las enfermeras de servicio en UCIN, ejecutan esta actividad de acuerdo con un plan de Medicamentos. Esta actividad requiere el conocimiento de la dosificación indicada para el tratamiento de cada paciente.

\section{Alimentos}

Este proceso registra la dosis de alimento en el moni- toreo de la hoja. Las dosis son indicadas por el médico en UCIN.

- Leer registro de tratamiento

Las enfermeras de servicio de UCIN ejecutan esta actividad de acuerdo con el plan de alimentación enteral y parenteral: en este caso las enfermeras requieren conocer la dosis prescrita por el médico para cada paciente.

\section{- Coloque el suministro de alimentos}

Registra la dosis de alimento para el paciente; Además se podrían registrar anotaciones o comentarios adicionales. 


\section{Evaluación de alta}

Este proceso registra la evaluación antes del alta: el resultado indica si el paciente es dado de alta o transferido a otra unidad (figura 10).

- Evaluación de registros

Después de la estabilización del paciente, el médico puede decidir dar de alta o transferir al paciente a otra unidad. En todos los casos debe realizarse un registro de evaluación final en el sistema y documentar las medidas adoptadas (Alta o transferencia).

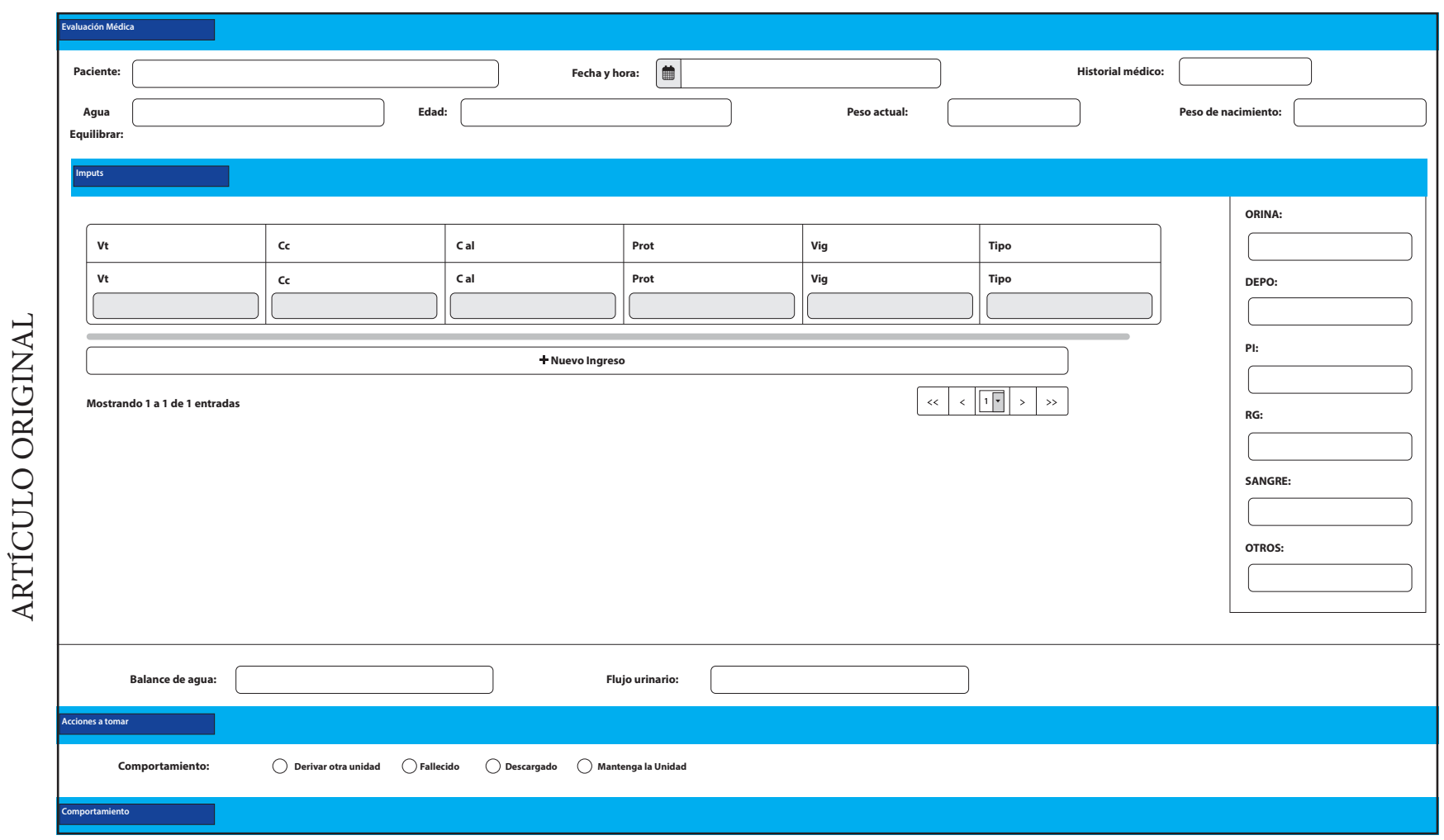

Figura 10. Evaluación de antes del alta.

\section{Registro de tratamiento}

Cuando el médico libera al paciente solicitar una transferencia a otra unidad, el tratamiento debe ser registrado.

\section{Tercera etapa: “Playback 2” - Integración}

La figura 9 muestra brevemente algunas consideraciones en términos de la arquitectura de la solución propuesta mHealth-BPM-UCIN. Para el proyecto por cuestiones de disponibilidad de la infraestructura se ha planteado una la arquitectura es compatible con la solución "IBM Business Process Manager"12.

\section{Diagrama de arquitectura global en UCIN}

Los componentes más importantes de la arquitectura son:

- Servidor de Directorios de Autenticación: Autenticación de Servidor (MS Active Directory) contra la cual autenticará y autorizará la ejecución de las tareas del personal médico y enfermeras. También contiene todos los grupos que se asignarán a tareas de proceso.
- Servidor de base de datos: Base de datos del servidor (MS-SQL) que admite la configuración de la plataforma IBM Business Process Manager y los procesos y tareas de datos (selección de datos, muestreo, etc.).

- Servidor de correo electrónico: Servidor de correo (MS Exchange) implicado en la emisión de notificaciones a los participantes (según las reglas) para presentar el desempeño de una tarea u otras.

- Servidores de plataformas BPM: El "IBM Business Process Manager" es responsable de la implementación y ejecución de procesos de negocio en UCIN. Presenta todas las tareas y responsabilidades a través del Portal Web. También cuenta con componentes de software que permiten la integración de la solución de software y la infraestructura de TI. Toda la integración se realiza a través de los servicios: estos servicios son necesarios para cumplir con los objetivos de una tarea en el proceso, ya sea en una entrada o salida de la transacción.

- Solución mHealth-BPM-UCIN (o UCIN / UCIN-BPM): 
Es el componente de software que representa el proceso empresarial implementado. Este componente consiste en un modelo de proceso ("Business Process Diagram: BPD") que utiliza la notación BPMN 2.0. Utiliza también otras extensiones tales como llamadas de servicios, servicios de presentación (GUI) a páginas web; También utiliza componentes basados en DOJO y/o Bootstrap. La definición de datos de negocio también se da en este componente. Estas extensiones son necesarias para gestionar las entradas y salidas de las tareas.

- Portal BPM Solución: Es el componente de software que implementa la solución del sitio web. El Portal participa de la autenticación de usuarios y tareas basadas en el servidor de autenticación. Permite la ejecución de tareas y la entrada de datos requerida para un proceso particular.

Componentes del diagrama mHealth-BPM - solución UCIN

El diagrama de la figura 10 representa los componentes internos de la solución mHealth-BPM-UCIN. Los elementos arquitectónicos más importantes son:

- BPM UCIN App: Representa la implementación del modelo de proceso empresarial (Business Process Diagram - BPD), también contiene definiciones de actividades empresariales, roles y responsabilidades de cada actividad, interfaces de usuario asociadas con la actividad y la entrada o salida para cada actividad.
- BPM UCIN Toolkit: Este elemento representa la biblioteca de tipos de datos, definiciones de servicio y plantillas de transacciones de entrada y salida. Esta información es utilizada por el proceso de negocio. La dependencia de los elementos "BPM UCIN App" es unidireccional y se maneja por el concepto de "instantánea" o versionado.

- BPM UCIN Services Facade: Este elemento es una biblioteca que contiene las interfaces con las llamadas a servicios externos en el proceso. Dado que este servicio no está asociado con un servicio de autobús en esta implementación, es el servicio más apropiado; Esta forma de diseño es la más apropiada.

- Aplicación de servicios de implementación de BPM UCIN: aplicación que implementa la coreografía para llamar a servicios externos; También permite la transformación de datos en información útil para cada servicio de tareas. La coreografía o transformación de datos se realiza mediante elementos denominados "Mediación"; Sus elementos controlan la integración.

- Paquete de servicios XX: son los paquetes que representan los servicios que están Ilamando a componentes externos. Estos servicios deben existir y estar disponibles para los componentes mHealth-BPM-UCIN. Los servicios pueden ser desarrollados por la institución u organizaciones públicas (ejemplo gobierno).

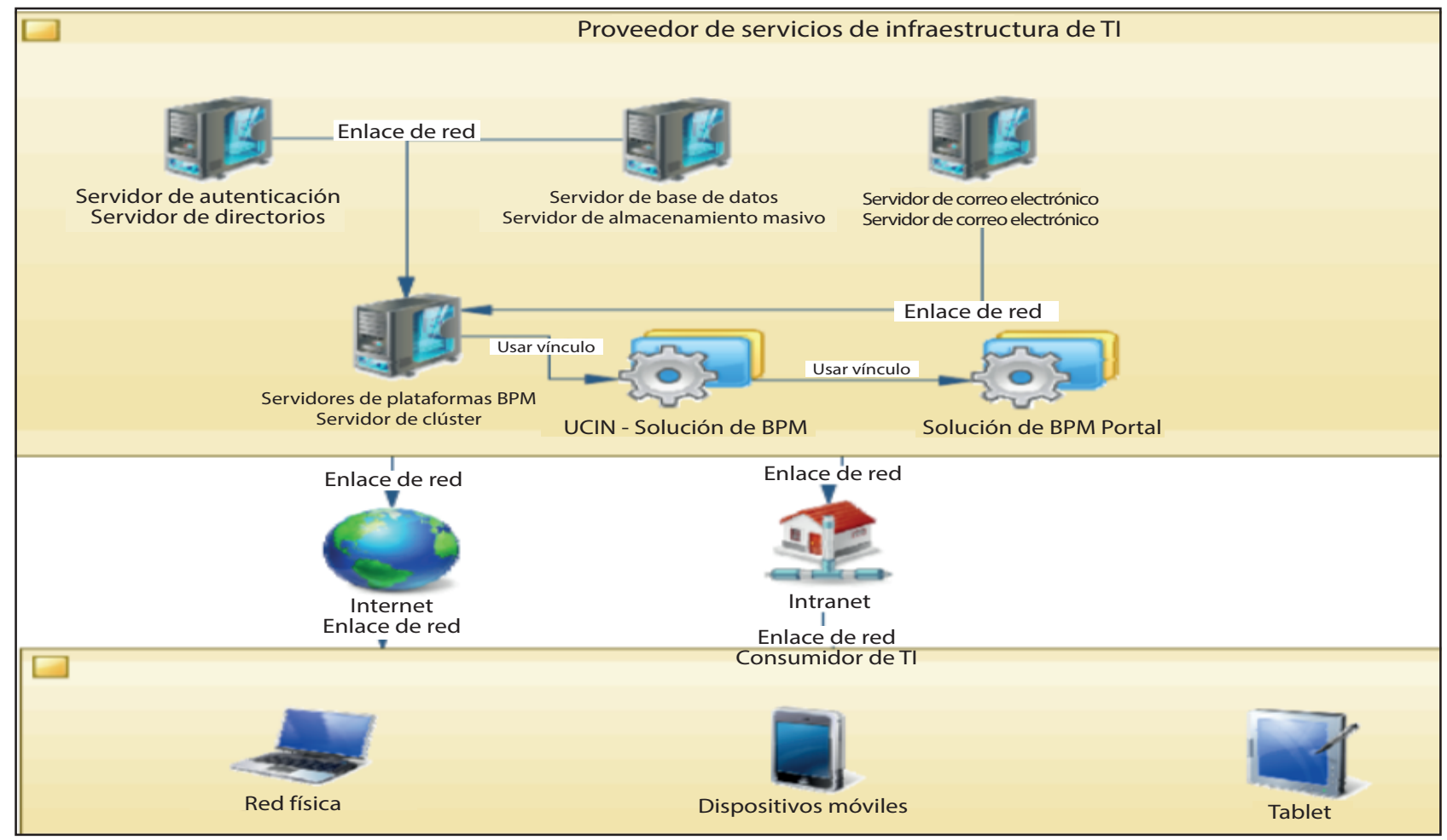

Figura 11. Diagrama de arquitectura global en UCIN. 


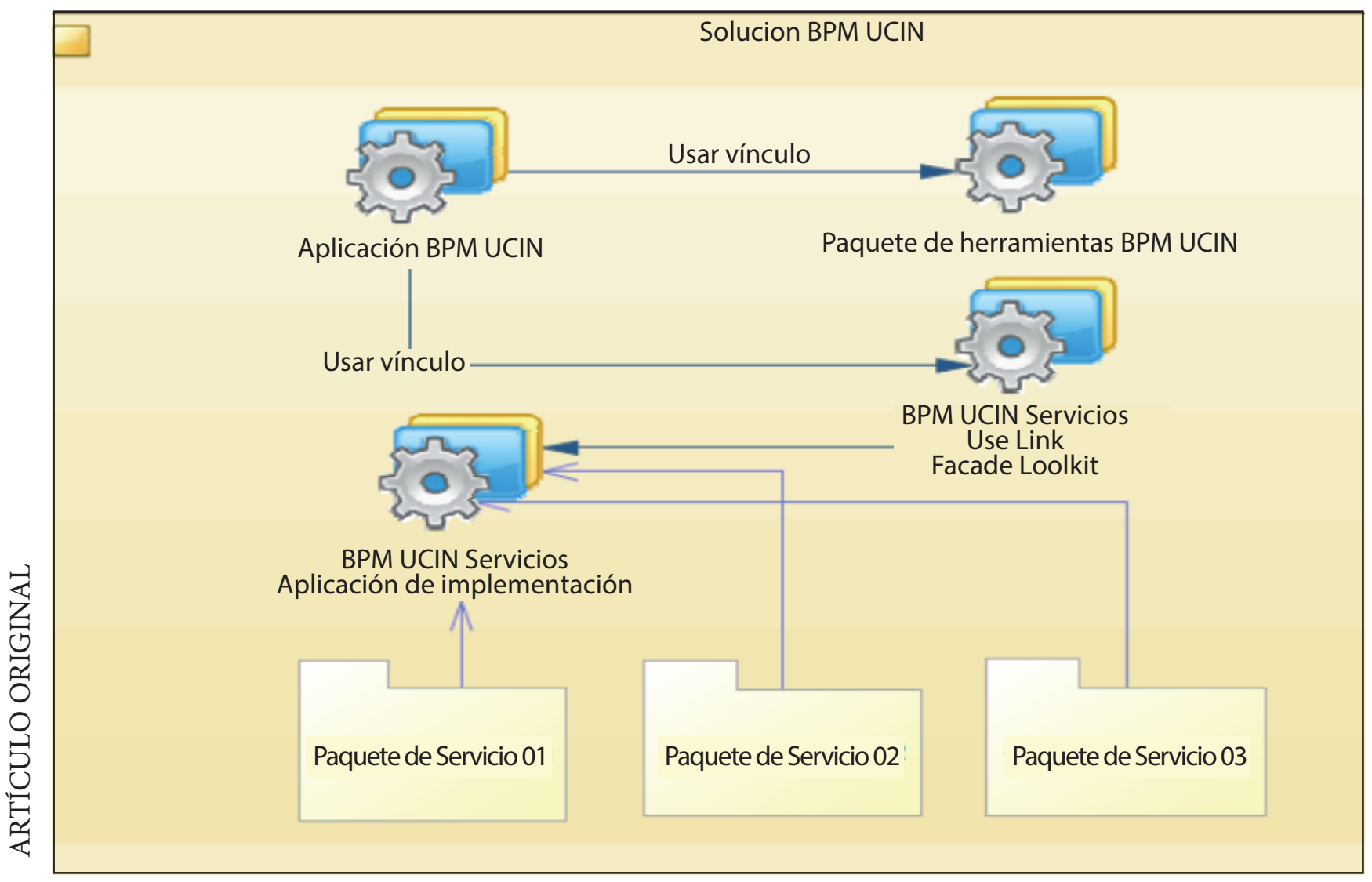

Figura 12. Componentes del diagrama BPM - UCIN Solution.

\section{Arquitectura de implementación de BPM}

La Figura 11 muestra detalles sobre ciertos componentes de arquitectura de la solución "IBM Business Process Manager" para UCIN:

- Base de datos de Process Center : Es un repositorio de datos de definiciones de procesos y todos los elementos involucrados, tales como: roles, actividades, usuarios, formularios, entrada de datos, salidas, etc. Las versiones de estos elementos se gestionan a tiempo como instantáneas. Una instantánea dada podría convertirse en ejecutable en un entorno de tiempo de ejecución en un servidor de procesos.

- Base de datos de Procesos de Servidores: Es el repositorio de datos de instantáneas desplegadas desde el "Centro de Procesos" y está compuesto de datos recopilados a nivel de ejecución de procesos de negocio. Toda la información sobre el tiempo de ejecución se almacena en la base de datos, incluyendo información sobre los estados de las variables de proceso utilizadas, entradas, salidas y datos necesarios para el seguimiento de todas las variables y elementos de un Performance de Proceso (KPI).

- IBM Process Center: Administra el repositorio de procesos y desempeña el rol de "Gestión de Procesos".
También incluye herramientas para procesos de modelado, pruebas unitarias y otras.

- IBM Process Server Runtime: Es el motor de aplicación para ejecutar procesos de negocio y contiene procesos de administración de aplicaciones "runtime"; También contiene aplicaciones para la integración de nivel de gestión. También tiene la "BPM Portal App", que es el estándar para la gestión de tareas.

- IBM Process Server Manager: Es la aplicación que supervisa una serie de "IBM Process Server Runtime"; Están a nivel de clustering y gestionan tareas y tareas de gestión de infraestructuras, como la implementación de una nueva instantánea, cambios en la configuración, etc.

- Servidor Web: Es el servidor web que permite presentar los servicios de la plataforma "IBM Business Process Manager"; Proporciona acceso a servidores que permiten una mejor seguridad y aislamiento. 


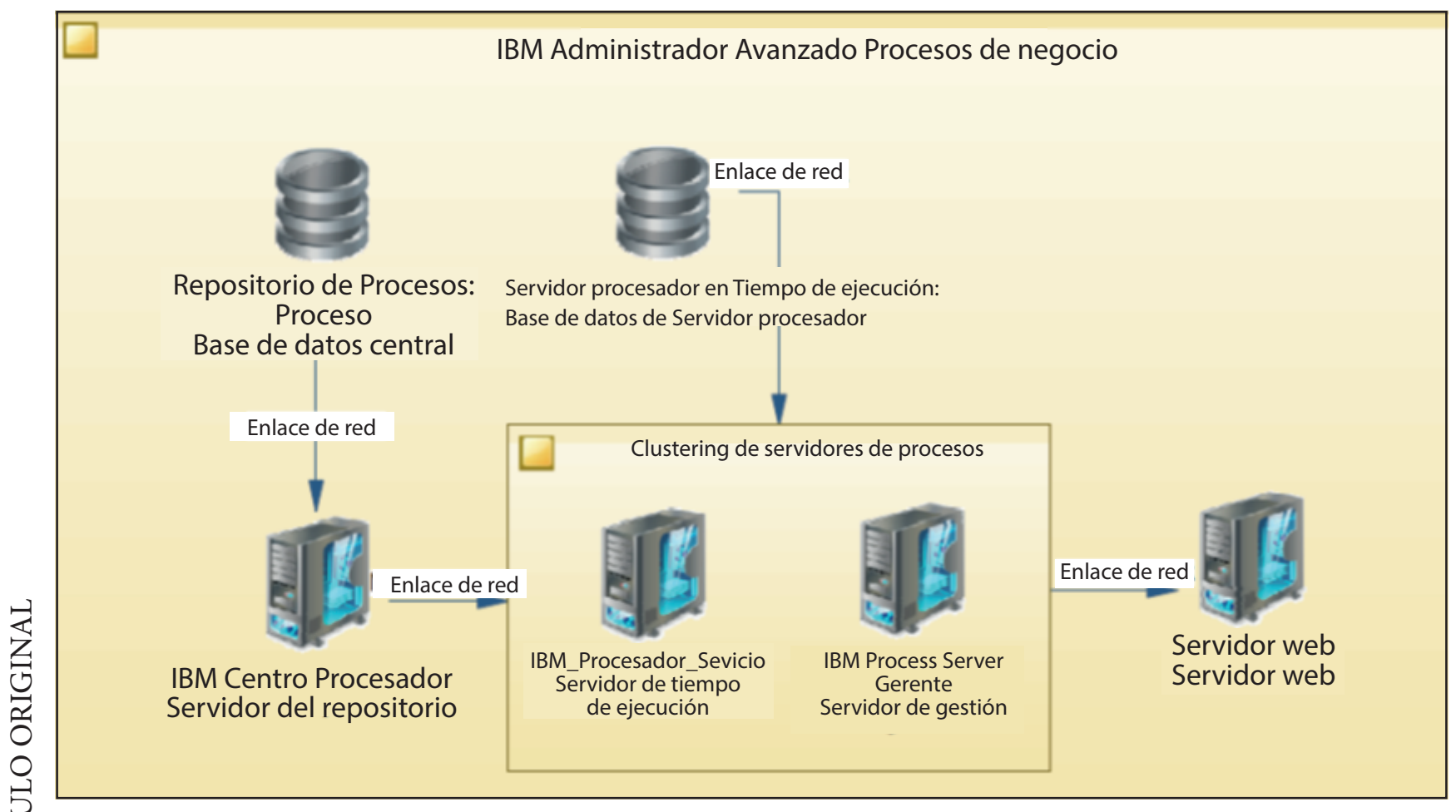

Figura 13. Arquitectura de implementación de BPM.

\section{Cuarta etapa: "Playback 3” - Refinar la entrega}

Actualmente, la solución mHealth-BPM-UCIN es un prototipo y las pruebas con la fase del usuario, los ajustes y las demostraciones deben hacerse al personal de UCIN. Los informes y las estadísticas deben adaptarse a los requisitos particulares de los usuarios. La solución se está ejecutando con datos históricos recogidos de múltiples fuentes.

Se planea realizar una carga histórica de los registros clínicos de algunos meses previos: consistirá en anotaciones manuales de los expedientes médicos en papel.

A continuación se presenta un ejemplo de la capacidad de la solución mHealth-BPM-UCIN para proporcionar estadísticas para cada proceso. En la figura 10 se muestra el indicador relativo al tiempo que lleva al médico el proceso "Admisión y Diagnóstico" y el proceso "Plan de Tratamiento".

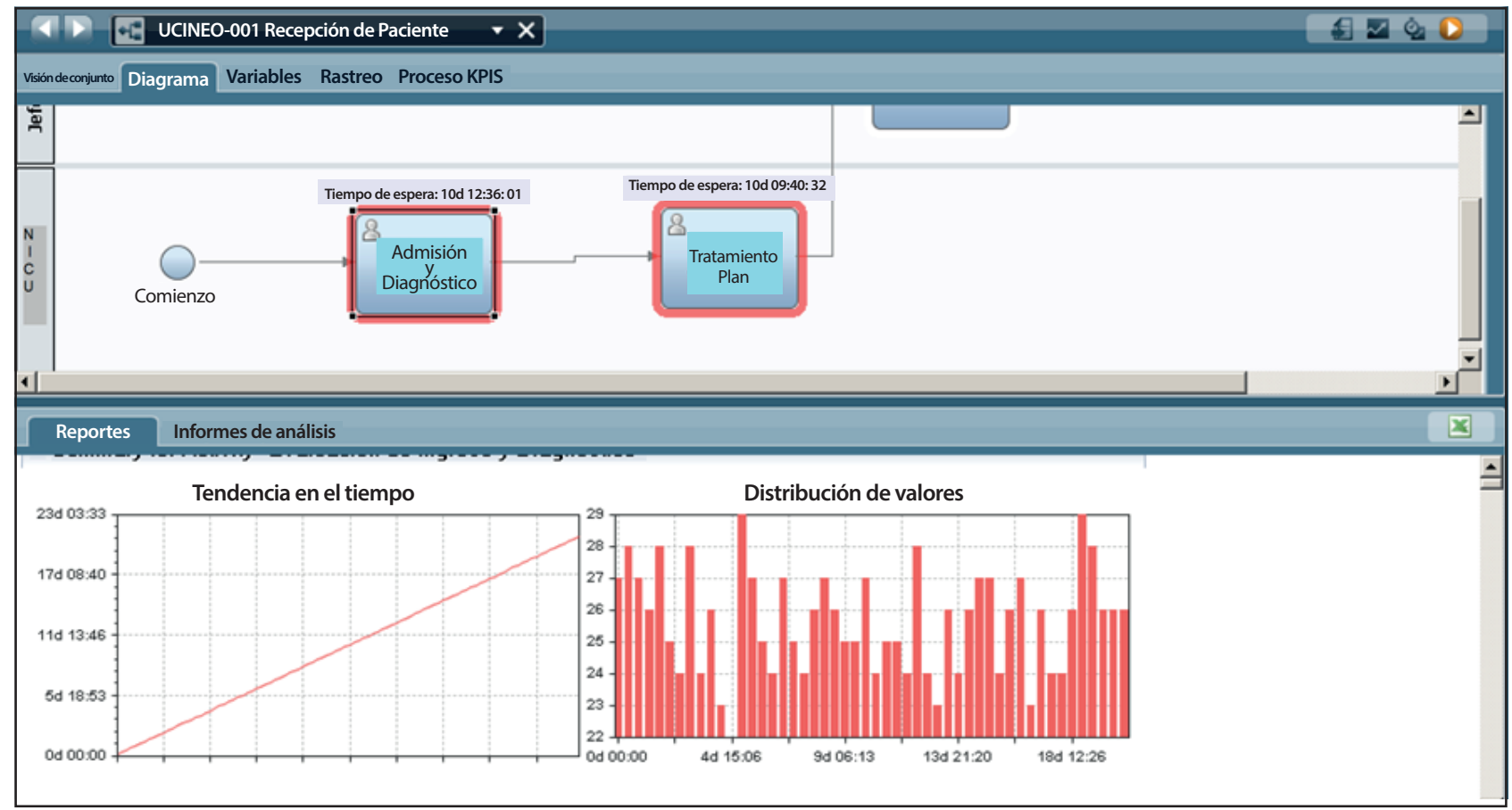

Figura 14. Rendimiento del proceso de admisión y diagnóstico. 


\section{CONCLUSIÓN}

Este capítulo ha presentado una solución basada en tecnología BPM web y móvil para abordar una realidad desafiante en una UCIN. Es bien sabido que, a veces, los hospitales ofrecen servicios de salud con recursos limitados en cuanto a equipos, profesionales, etc. Además, las áreas de emergencia y hospitalización a menudo están sobrecargadas de pacientes, lo que hace que el suministro de servicios de salud sea muy exigente.

La meta del proyecto fue diseñar un Sistema de Información sobre una plataforma móvil y web con procesos mejorados y automatizados para proveer al personal médico y de enfermería, un nivel adecuado de seguridad y acceso.

Como se ha mostrado la aplicación tiene la capacidad de registrar y monitorear los resultados de procedimientos, tratamientos médicos, los medicamentos prescritos, la información básica sobre la madre, las condiciones de parto promedio, el seguimiento de los resultados de los pacientes en la unidad, el cálculo de dosis de fármaco a los pacientes, la aprobación y la asignación de personal a los pacientes, el número de pacientes tratados y la duración de la estancia.

Se seleccionó la metodología BPM de "Playback" para implementar mejoras, gestionar procesos de negocio y crear interfaces Web y Móviles, conexiones de bases de datos, indicadores, arquitecturas y otros. La metodología "Playback" se basó en un proceso de implementación iterativa.

El proyecto fue diseñado e implementado con la solución "IBM Business Process Manager". La solución mHealth-BPM-UCIN está en un prototipo y una fase de prueba en la UCIN.

\section{Financiamiento: Autofinanciado.}

Conflicto de interés: Los autores declaran no tener conflictos de interés en la publicación de este artículo.

Recibido: 15 de Junio de 2016

Aprobado: 19 de Julio de 2016

\section{REFERENCIAS BIBLIOGRÁFICAS}

1. Brocke V, \& Rosemann M. Handbook on Business Process Management Berlin: Springer 2010.

2. Brown P, Kelly J, \& Querusio D. Toward a Healthcare Business-Process Reference Model. In IEEE Computer Society. 2011.

3. Dyer L, Flournoy H, Lehmann I, Lipof G, Osmani F, Parrott D. Scaling BPM Adoption From Project to Program with IBM Business Process Manager; IBM Redbook. 2012.

4. Hamou A, O'Brien J, Guy S, \& Gwadry-Sridhar F. InfoFrame - An Intelligent Informatics Data Collection Framework. Paper presented at the 2011 24th International Symposium on Computer-Based Medical Systems. 2011.

5. McGregor C, Bryan G, Curry J, \& Tracy M. The e-Baby Data Warehouse: A Case Study. Paper presented at the Proceedings of the 35th Hawaii International Conference on System Sciences. 2002.

6. MINSA. Norma Técnica de los Servicios de Cuidados Intensivos de Hospitales del Sector Salud In N. N. M. D. V. Perú. (Ed.): Ministerio de Salud del Peru. 2004.
7. OMG.. Business Process Model And Notation (BPMN) Version 2.0, from http://www.omg.org/spec/BPMN/2.0/. 2011.

8. Portela, F., Vilas-Boas, M., Santos, M. F., Abelha, A., Machado, J., Cabral, A., \& Aragao, I. Electronic Health Records in the Emergency Room. 195200. doi: 10.1109/icis.2010.98.

9. Ray, S., Dogra, D., Bhattacharya, S., Saha, B., Biswas, A., Majumdar, A., Bhattacharya, S. D. A Web Enabled Health Information System for the Neonatal Intensive Care Unit (UCIN). 451-458. doi: 10.1109/services.2011.38

10. Seguel C. Dirección General de Aguas MOP Proyecto: “Optimización de Recursos con el apoyo de la Gestión por Procesos". Chile: Ministerio de Obras Publicas. 2013.

11. Simmons, S. BPM Voices: Evaluating BPM applications: BPM design reviews and Rubik's Cubes. IBM Business Process Management Journal. 2013.

12. Underdahl. IBM Business Process Management: Wiley Publishing Inc. 2011. 